\title{
Nanocrystalline diamond microelectrode arrays fabricated on sapphire technology for high-time resolution of quantal catecholamine secretion from chromaffin cells
}

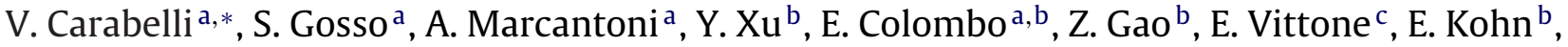 \\ A. Pasquarelli ${ }^{\mathrm{b}}$, E. Carbone ${ }^{\mathrm{a}}$ \\ a Department of Neuroscience, NIS Centre of Excellence University of Torino, Corso Raffaello 30, 10125 Torino, Italy \\ ${ }^{\mathrm{b}}$ Institute of Electron Devices and Circuits, Ulm University, 89081 Ulm, Germany \\ ${ }^{c}$ Department of Experimental Physics, NIS Centre of Excellence University of Torino, 10125 Torino, Italy
}

\section{A R T I C L E I N F O}

\section{Article history:}

Received 5 February 2010

Received in revised form 15 April 2010

Accepted 10 May 2010

Available online 19 May 2010

\section{Keywords:}

Nanocrystalline diamond

Chromaffin cell

Amperometry

$\mathrm{Ca}^{2+}$-dependent exocytosis

Quantal secretory event

\begin{abstract}
A B S T R A C T
The quantal release of oxidizable molecules can be successfully monitored by means of polarized carbon fiber microelectrodes (CFEs) positioned in close proximity to the cell membrane. To partially overcome certain CFE limitations, mainly related to their low spatial resolution and lack of optical transparency, we developed a planar boron-doped nanocrystalline diamond (NCD) prototype, grown on a transparent sapphire wafer. Responsiveness to applied catecholamines as well as the electrochemical and optical properties of the NCD-based device were first characterized by cyclic voltammetry and optical transmittance measurements. By stimulating chromaffin cells positioned on the device with external $\mathrm{KCl}$, well-resolved quantal exocytotic events could be detected either from one NCD microelectrode, or simultaneously from an array of four microelectrodes, indicating that the chip is able to monitor secretory events (amperometric spikes) from a number of isolated chromaffin cells. Spikes detected by the planar NCD device had comparable amplitudes, kinetics and vesicle diameter distributions as those measured by conventional CFEs from the same chromaffin cell.
\end{abstract}

(c) 2010 Elsevier B.V. All rights reserved.

\section{Introduction}

In neurons and neuroendocrine cells, neurotransmitters are packed in intracellular vesicles of 50-200 nm diameter and released in the form of "quanta" into the extracellular space after vesicle fusion with the plasma membrane (exocytosis). The quantal nature of neurotransmitter release is at the basis of synaptic functioning and has been investigated since the early 1990s by means of carbon fiber microelectrodes (CFEs) (Wightman et al., 1991). When applied to detect exocytotic events in isolated chromaffin cells, CFEs can provide key information on the formation of the fusion pore and the kinetics of single secretory events with sub-millisecond time resolution (Chow et al., 1992). The technique, however, has some major restrictions. Monitoring quantal secretory events is limited to one cell for each recording and there is no spatial resolution of vesicle distribution below the area of carbon fiber tip.

Following well-established methods used for studying neural networks and acute slices, in this work we adopted a simplified planar microelectrode configuration for recording quantal secretory events. Recently, other planar amperometric devices based on var-

\footnotetext{
* Corresponding author. Tel.: +39 011 6708488; fax: +39 0116708174

E-mail address: valentina.carabelli@unito.it (V. Carabelli).
}

ious technologies, e.g. platinum, gold, indium-doped tin oxide (ITO) and nitrogen-doped diamond-like carbon (DLC:N) deposited on glass and silicon layers have been shown to monitor well-resolved quantal amperometric events (Dias et al., 2002; Chen et al., 2003, 2008; Hafez et al., 2005; Parpura, 2005; Cui et al., 2006; Gao et al., 2008). Among these technologies, ITO is the most interesting one for producing transparent electrodes and wires (Guillen and Herrero, 2005). It is more transparent than boron-doped NCD and works nicely in the anodic regime (Sun and Gillis, 2006). However, ITO shows a lower chemical stability, especially at acidic $\mathrm{pH}$ and cathodic potentials, and a narrower potential window with respect to the $\sim 3 \mathrm{~V}$ of boron-doped NCD electrodes (Sun and Gillis, 2006; Chen et al., 2008; Sen et al., 2009).

Here we describe a cost-effective long-life device that can potentially be exploited in a wide variety of amperometric measurements, thanks to the superior properties of diamond technology. To this purpose we developed a chip prototype made using nanocrystalline diamond thin-film technology on sapphire, patterned with four boron-doped microelectrodes. Diamond is optically transparent, highly biocompatible, chemically inert and can exhibit a quasi-metallic conductivity when properly doped (Show et al., 2003) or functionalised (Ariano et al., 2009). Moreover, the high corrosion resistance of the NCD electrodes ensures a long-term reliability not easily achievable with other elec- 

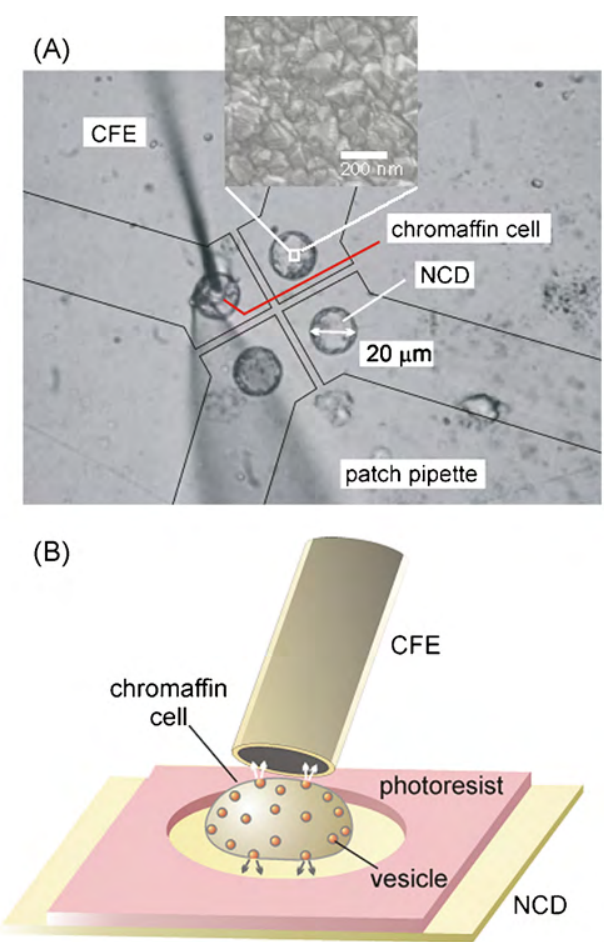

(C)

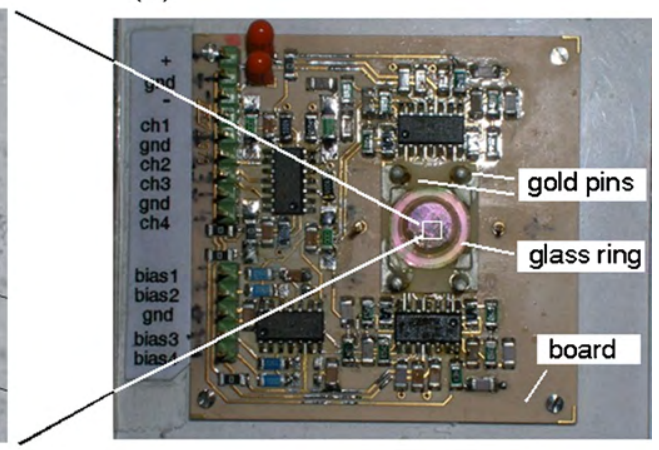

(D)

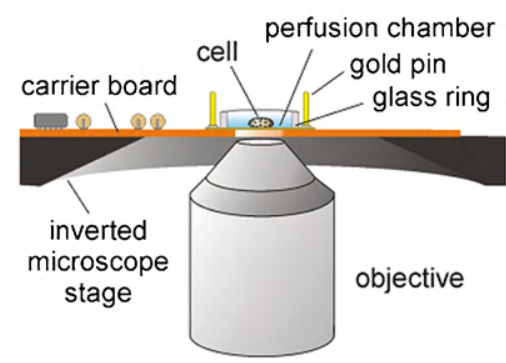



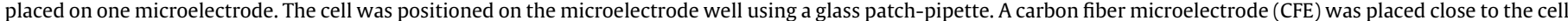

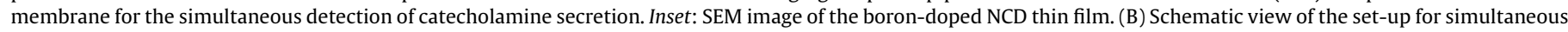

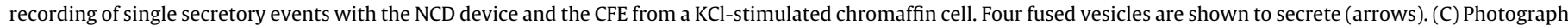

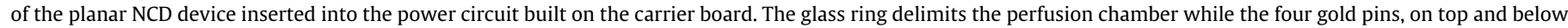

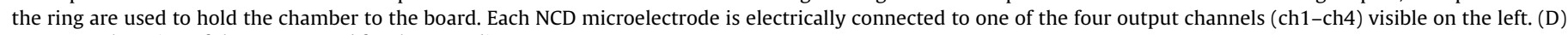
Transversal section of the set-up used for the recordings.

trode materials. Stable covalent functionalisations and different terminations $(\mathrm{H}, \mathrm{NH}, \mathrm{O}, \mathrm{F})$ can be easily obtained on the NCD electrode surface. This, together with the optical transparency, make boron-doped NCD on sapphire a promising material to combine electrochemical and fluorescence detection for biosensing applications (Zhou and Zhi, 2009; Luong et al., 2009). Boron-doped diamond thin films deposited on a conical Pt wire have been already successfully used for low time resolution amperometric monitoring of NE release from sympathetic nerves innervating a large area of the mesenteric artery (Park et al., 2006).

Taking advantage of the unique properties of NCD electrodes on sapphire, we measured secretory quantal events from cultured chromaffin cells, a model of sympathetic postganglionic neurons that stores large quantities of catecholamines (up to $1 \mathrm{M}$ ) inside the chromaffin granules. Quantal secretory signals induced by external $\mathrm{KCl}$ stimulation had comparable amplitude and time courses as those recorded with CFEs. The new boron-doped NCD device has adequate space-time resolution and the sensitivity to measure the rapid release of catecholamines simultaneously from different cells in the form of single amperometric events. The planar configuration of the new solid-state device greatly increases the number of electrochemical applications, allowing the construction of multielectrode arrays for the simultaneous recordings of amperometric and fluorescence signals from different cells or, if required, from different areas of the same cell.

\section{Materials and methods}

\subsection{Device fabrication and design}

Starting from a single crystal double-polished $330 \mu \mathrm{m}$ thick sapphire wafer, a Si-based nucleation layer was first deposited by PECVD (Plasma Enhanced Chemical Vapor Deposition). The formation of nanodiamond nuclei was driven by Bias Enhanced Nucleation (BEN) in a HFCVD (Hot Filament CVD) system (Janischowsky et al., 2003), while processing the substrate at a temperature of $800^{\circ} \mathrm{C}$ in an atmosphere of $0.75 \% \mathrm{CH}_{4}$ in $\mathrm{H}_{2}$ at a pressure of $1.5 \mathrm{kPa}$, yielding a nucleation density of approximately $3 \times 10^{10} \mathrm{~cm}^{-2}$. Onto this substrate a $200 \mathrm{~nm}$ layer of intrinsic NCD was grown, keeping the substrate at a temperature of $\sim 740^{\circ} \mathrm{C}$ in a gas mixture of $0.3 \% \mathrm{CH}_{4}$ in $\mathrm{H}_{2}$, at a pressure $2.5 \mathrm{kPa}$. The last step consisted in growing a quasi-metallic boron doped top layer of $350 \mathrm{~nm}$ thickness adding trimethyl borate $1.5 \%$ in $\mathrm{H}_{2}$ under the same parameters used for intrinsic growth. The whole process described above is computer controlled and requires minimal manual intervention; furthermore the use of a palladium membrane for hydrogen filtration allows the use of low-purity grade gas precursors.

The microelectrode array was realized using a simple threemasks microfabrication procedure, first by patterning the sensitive areas with a gap distance of $\sim 4 \mu \mathrm{m}$ by optical lithography and then dry etching in an $\mathrm{O}_{2} / \mathrm{Ar}$ plasma down to the sapphire substrate (see Fig. 1A). Ohmic contacts have then been realized by Ti/Au deposition at the corners of the $10 \mathrm{~mm} \times 10 \mathrm{~mm}$ device. The electrodes underwent a conditioning process to remove the residual graphitic phases at the grain boundaries of the nanocrystalline structure and produce an oxygen terminated hydrophilic surface, by exposure for 5 min to oxygen plasma and then by applying an anodic treatment in the oxygen evolution range in $0.1 \mathrm{M} \mathrm{KOH}$ at $1.8 \mathrm{~V}$ versus SCE (Saturated Calomel Electrode) for $3 \mathrm{~min}$, resulting in a total charge transfer of $0.3 \mathrm{C} / \mathrm{cm}^{2}$. The capacitance of the electrodes as evaluated by impedance spectroscopy measurements at $\mathrm{pH} 7$ and $650 \mathrm{mV}$ was $\sim 3 \mu \mathrm{F} / \mathrm{cm}^{2}$. 
The electrochemically active areas of the microelectrode array were finally defined by patterning an SU-8 passivation layer with four circular openings of $20 \mu \mathrm{m}$ in diameter onto the chip surface, as shown in Fig. 1A and B (SU-8 is an epoxy-based, highly chemically inert photoresist). Before packaging, the device was electrically characterized using an HP4145B semiconductor parameter analyzer, to assess both the electrodes resistance and the inter-electrodes insulation. Typical measured values were $20-30 \mathrm{k} \Omega$ for the resistance and approximately $1 \mathrm{~T} \Omega$ for the insulation.

The device was then assembled onto a carrier board (Roger R4003, to preserve the dielectric properties), contacted with silver paste and finally provided with a $200 \mu$ l glass ring as perfusion chamber. This final set-up is our preferred version, because it allows the concurrent use of traditional patch-clamp pipette microelectrodes for recording/stimulation of electrophysiological activity of a single cell. Yet the planar design may also allow the use of a microfluidic system for cell measurement automation. The complete assembly, including the "home-made" board with the signal conditioning electronics and the final mounting on the microscope stage, is shown in Fig. 1C and D.

Signal acquisition was performed by means of National Instruments hardware and software, working with 16 bit resolution at a sampling rate of $4000 \mathrm{~Hz}$ per channel and a bandwidth from DC to $1000 \mathrm{~Hz}$. The overall noise level was about $5 \mathrm{pA}$ peak-to-peak, i.e. substantially the same noise level obtained from the CF set-up. More recently we begun focusing on the noise issues and could already improve by one order of magnitude the signal-to-noise ratio, using a more accurate hardware assembly and a better tuning of the working parameters. On the basis of these tests, we believe that the intrinsic noise floor of the diamond electrodes is not yet reached and further improvements are likely.

\subsection{Isolation and culture of mouse chromaffin cells}

All experiments have been carried out in accordance with the directive 86/609/EEC for animal experiments. Chromaffin cells of the adrenal medulla were obtained from young (1-3 months) C57BL/6N mice killed by cervical dislocation, and cultured as previously described (Marcantoni et al., 2009). Medulla digestion was achieved for $60 \mathrm{~min}$ at $37^{\circ} \mathrm{C}$ in a DMEM's solution containing (in $\mathrm{mM}$ ): $1.5 \mathrm{~L}$-cysteine, $1 \mathrm{CaCl}_{2}, 0.5$ EDTA and $20 \mathrm{U} / \mathrm{ml}$ of papain (Worthington Biochemical Corp., Lakewood, NJ). Cells suspension was then centrifuged for $5 \mathrm{~min}$ at $900 \mathrm{rpm}$, washed two times with a Locke's solution containing $1 \mathrm{mM} \mathrm{CaCl}_{2}$ and $10 \mathrm{mg} / \mathrm{ml}$ bovine serum albumin (BSA). Cells were then resuspended in $2 \mathrm{ml}$ DMEM supplemented with $15 \%$ fetal calf serum (FCS) and plated in four-well plastic dishes.

Cells were cultured either adhered to the dishes using poly-Lornithine $(0.5 \mathrm{mg} / \mathrm{ml})$ and laminin $(10 \mu \mathrm{g} / \mathrm{ml}$ in $\mathrm{L}-15$ carbonate) as substrates or not adhered (floating) using $5 \%$ BSA. In both cases, after $1 \mathrm{~h}, 1.8 \mathrm{ml}$ of DMEM supplemented with $15 \%$ fetal calf serum (FCS) (Invitrogen, Grand Island, NY), $50 \mathrm{IU} / \mathrm{ml}$ penicillin, and $50 \mu \mathrm{g} / \mathrm{ml}$ streptomycin (Invitrogen) was added to the wells. Cells were then incubated at $37^{\circ} \mathrm{C}$ in a water-saturated atmosphere with $5 \% \mathrm{CO}_{2}$ and used within 2-4 days after plating.

\subsection{Amperometric detection of exocytosis from chromaffin cells}

For amperometric recordings using the planar NCD microelectrode array, chromaffin cells were removed from BSA-treated dishes and centrifuged for $4 \mathrm{~min}$ at $900 \mathrm{rpm}$. The pellet was resuspended in a $\mathrm{KCl}$-free solution and $30 \mu \mathrm{l}$ of the cell suspension was loaded into the device reservoir. Cells were allowed to settle for few minutes before recordings. If needed, cells were positioned on the microelectrodes with a glass patch-clamp pipette controlled by a motor-driven micromanipulator under visual inspection through the inverted microscope (Fig. 1A). Exocytosis was stimulated by applying $100 \mu \mathrm{l}$ of the $\mathrm{KCl}$-enriched solution. In a subset of experiments, exocytosis was simultaneously monitored by the NCD device and a carbon fiber microelectrode (CFE). CFEs were purchased from ALA Scientific Instruments Inc. (Westbury, NY, USA), and cut at $45^{\circ}$ before use. CFEs were polarized at $+650 \mathrm{mV}$ and carefully positioned adjacent to the cell membrane using motor-driven micromanipulators (Fig. 1B).

For detecting catecholamine secretion by means of CFEs, chromaffin cells were plated in plastic dishes pre-treated with polyL-ornithine $(0.5 \mathrm{mg} / \mathrm{ml})$ and laminin $(10 \mu \mathrm{g} / \mathrm{ml}$ in L-15 carbonate). Before stimulation, cells were bathed into an extracellular $\mathrm{KCl}$-free solution containing (mM): $130 \mathrm{NaCl}, 2 \mathrm{MgCl}_{2}, 10$ HEPES, 10 glucose, $10 \mathrm{CaCl}_{2}$, in order to hyperpolarize the cell membrane and minimize resting exocytosis. Then cells were perfused with a $\mathrm{KCl}$-enriched solution, containing (mM): $100 \mathrm{NaCl}, 2 \mathrm{MgCl}_{2}, 10 \mathrm{HEPES}, 10$ glucose, $30 \mathrm{KCl}, 10 \mathrm{CaCl}_{2}$. Recordings of amperometric signals (spikes) were performed using an EPC-10 amplifier (HEKA). Signals were sampled at $4 \mathrm{kHz}$, low-pass-filtered at $1 \mathrm{kHz}$ and monitored during 2-min recordings. Data analysis of amperometric spikes was performed by means of Igor macros, as previously described (Carabelli et al., 2007).

\subsection{Voltage-clamp recordings}

$\mathrm{Ca}^{2+}$ currents and the depolarization-evoked capacitance increases were measured in the perforated-patch configuration, by means of an EPC-10 patch-clamp amplifier (HEKA Elektronik, Lambrecht, Germany). The extracellular solution contained (mM): 130 $\mathrm{NaCl}, 2 \mathrm{MgCl}_{2}, 10$ HEPES, 10 glucose, and $10 \mathrm{CaCl}_{2}$ (pH 7.3 with $\mathrm{NaOH}$ ). Patch-pipettes were made of thin borosilicate glass (Kimax 51; Witz Scientific, Holland, OH, USA) and filled with an internal solution containing (in mM): $135 \mathrm{CsMeSO}_{3}, 8 \mathrm{NaCl}, 2 \mathrm{MgCl}_{2}, 20$ HEPES, pH 7.3, with CsOH plus amphotericin B. $\mathrm{Ca}^{2+}$ currents were sampled at $10 \mathrm{kHz}$ and filtered at $2 \mathrm{kHz}$. Depolarization-evoked exocytosis was measured as membrane capacitance increases by applying a sinusoidal wave function on the holding potential, as previously described (Carabelli et al., 2007; Marcantoni et al., 2009). Fast capacitive transients due to depolarizing pulses were minimized online by the patch-clamp analog compensation. Series resistance was compensated by $80 \%$ and monitored during the experiment.

\section{Results and discussion}

\subsection{Transparency of the NCD-based device}

Due to their intrinsic transparency, NCD microelectrodes should facilitate simultaneous detection of optical and electrochemical signals. For this reason we investigated the spectral transmittance of the complete stack (fully processed device) in the wavelength range between 350 and $900 \mathrm{~nm}$. It is easy to predict any complex optical behavior of the device stack due to the different optical properties of the individual layers. In fact, as extensively discussed elsewhere (Gao et al., 2010), the heavily boron-doped electrode layer, the undoped diamond support layer, the Si-based nucleation interlayer and even the SU-8 photoresist used for passivation, in addition to the scattering behavior due to the nanocrystalline structure of the film together with the little yet non-negligible sp2 content at the grain boundaries, cause composite optical absorptions and interferences. The overall transmittance is shown in Fig. 2A. Worth mentioning is the systematic decrease of the average transmittance as the wavelength decreases (Gao et al., 2010). This feature is superimposed on the interference fringes due to multi- 

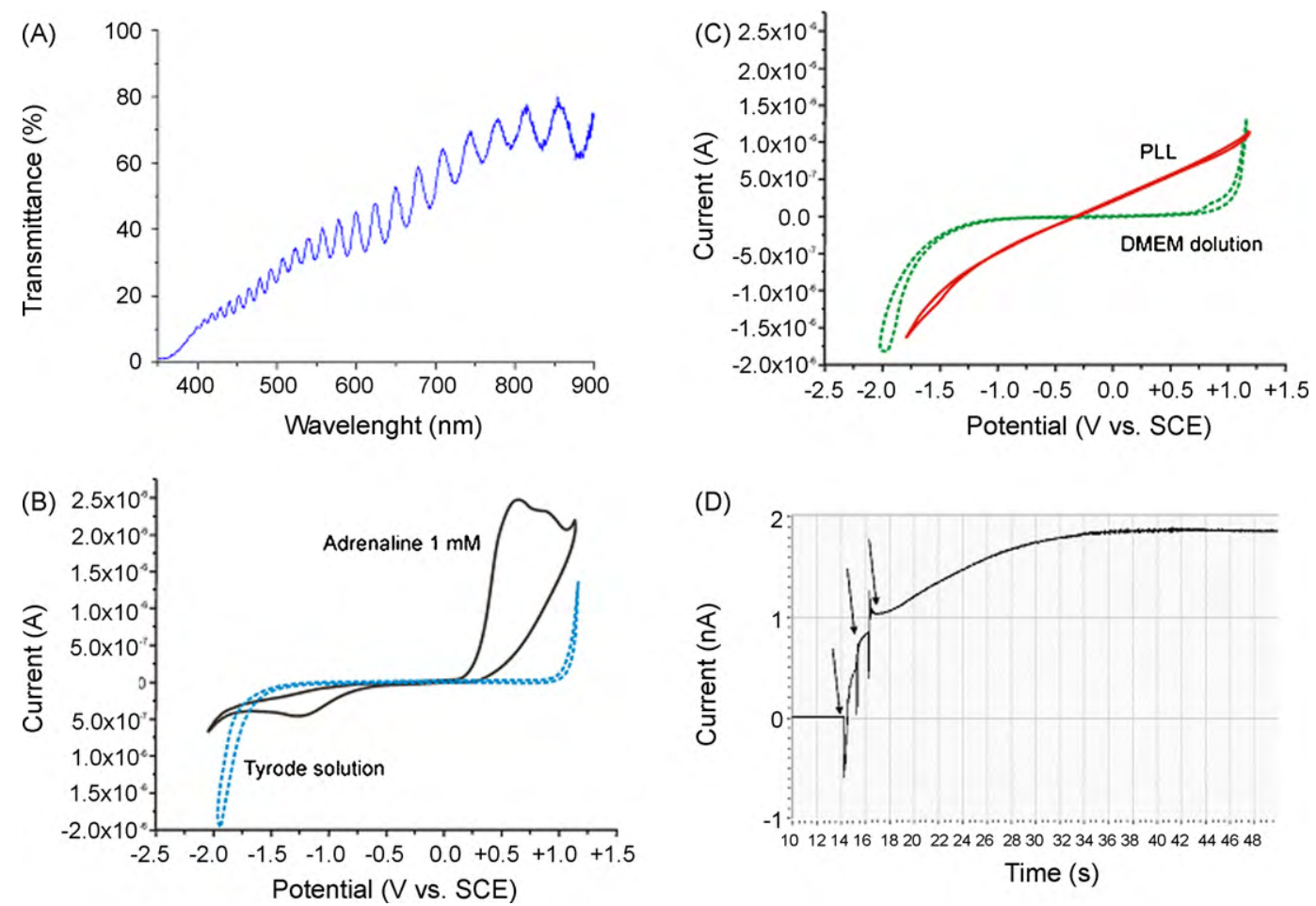



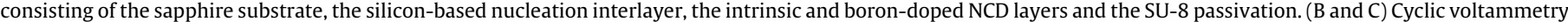

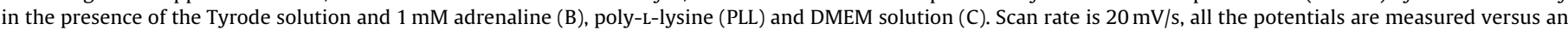
$\mathrm{Ag} / \mathrm{AgCl}$ reference electrode. (D) Response of a boron-doped NCD microelectrode to three consecutive drops (arrows) of $1 \mathrm{mM}$ adrenaline solution.

beam interference occurring at the interfaces of the thin-film stack (Gao et al., 2009).

Looking more carefully at the spectrum of transmittance it is evident that the NCD-based device is suitable for biofluorescence applications. In fact, in the wavelengths range between 500 and $600 \mathrm{~nm}$ where typical fluorophores, such as Fluo-3 and Texas Red are used to monitor intracellular $\mathrm{Ca}^{2+}$ (Titus et al., 1982; Minta et al., 1989), the device shows a transparency of $30-40 \%$ on the passivated surface (Fig. 2A), and even higher values (40-60\%) in the area uncovered by the photoresist, where the amperometric measurements take place (Gao et al., 2010). Considering a typical dynamic range of $10^{4}$ for the laser induced fluorescence (LIF) detection method, it is possible to conclude that in spite of the marked absorption in the blue-violet region, the average transmittance of the device in the visible range allows fluorescence analysis with a residual dynamic range larger than three orders of magnitude.

\subsection{Cyclic voltammetry on NCD microelectrodes}

The cell culture environment contains several ion species and molecules, ranging from simple electrolytes to complex nutrients, antibiotics and growth factors. These components could interfere with the targeted amperometric detection and spoil both sensitivity and signal-to-noise ratio. It was therefore of primary importance to investigate not only the dark current and the hydrolysis potential window of the electrode in distilled water, but also the redox properties of the saline solution, culture medium and other protein-like adhesion substrates used. To this purpose, four cyclic voltammetric measurements were undertaken to characterize the NCD microelectrode material in the working environments (see Fig. 2B and C): (i) NCD in Tyrode saline solution ( $\mathrm{pH} 7.4$ ), which showed neither a redox activity within the $\sim 3 \mathrm{~V}$ hydrolysis window nor a degradation of the $1 \mathrm{nA} / \mathrm{mm}^{2}$ dark current, (ii) NCD in DMEM solution that exhibited little oxidation activity at $+900 \mathrm{mV}$, (iii) NCD in Tyrode solution with $1 \mathrm{mM}$ adrenaline to assess the catalytic properties of the electrode material and determine the optimal biasing voltage. This was at around $+650 \mathrm{mV}$, where the oxidation current reached its maximum, (iv) finally, we tested the effects of poly-L-lysine (PLL) to evaluate whether this commonly used cell adhesion layer could modify the electrode properties. In the presence of PLL the native redox electrode responded almost linearly with a large background current that was about $30 \%$ of that measured with adrenaline at $+650 \mathrm{mV}$. To better understand this latter result, we also checked the bulk conductivity of Tyrode solution with and without PLL using a pair of non-polarizable $\mathrm{Ag} / \mathrm{AgCl}$ electrodes, but we found no significant deviation from the normal value of $14 \pm 1 \mathrm{mS} / \mathrm{cm}$. It appears evident that the polycathionic structure of PLL modifies the electrochemical properties of the solid/liquid junction and therefore this adhesion substrate is not compatible with amperometric measurements performed with NCD planar electrodes. Because of this we avoided using PLL-treated NCD devices.

All the above results clearly show the wide application potential of the being presented device, which extends not only to anodic overpotentials, but has also a remarkably wide working range for electrochemical reduction reactions in the cathodic regime, where other transparent materials degrade rapidly.

\subsection{NCD microelectrode responses to adrenaline solutions}

In a preliminary series of experiments we also tested the electrochemical responsiveness of the NCD on sapphire to oxidizable molecules by direct application of adrenaline $(1 \mathrm{mM})$. For amperometric recordings, a constant $+650 \mathrm{mV}$ potential (with respect to the $\mathrm{Ag} / \mathrm{AgCl}$ reference electrode) was applied to the microelectrode. The NCD microelectrode array was initially bathed with a standard Tyrode solution (control). Addition of three consecutive drops of adrenaline (as indicated by the arrows in Fig. 2D) caused the generation of oxidation currents which reached maximum amplitude 
(A)

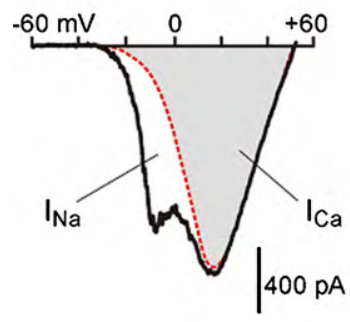

(B)
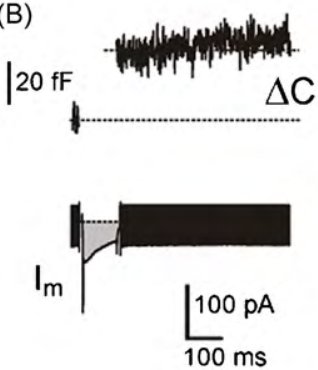

(C)



$I_{m}$

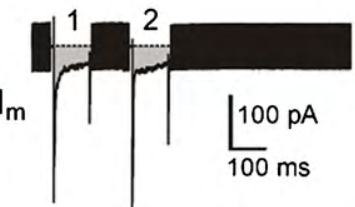



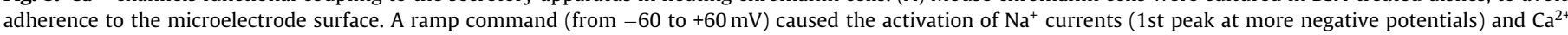

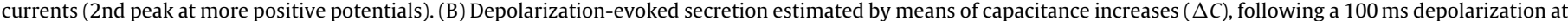

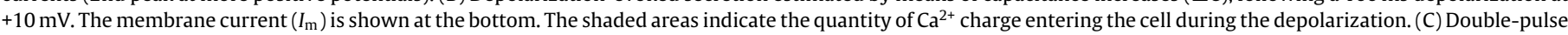
protocol used for measuring the size of the readily releasable pool (RRP).

( $1.8 \mathrm{nA})$ within approximately $30 \mathrm{~s}$. The upward drifts in the current over time are due to the diffusion of adrenaline molecules reaching the electrode. The current persisted at its steady-state value for over $60 \mathrm{~s}$, thus confirming the sensitivity of the NCD electrode on sapphire to oxidizable molecules and the presence of a negligible dark current. As a control, we also tested the effects of adrenaline-free Tyrode solutions and had no detectable responses, thus excluding the possibility that the current changes of Fig. 2B were caused by solution-exchange artifacts.

\section{4. $\mathrm{Ca}^{2+}$-dependent secretion measured in floating chromaffin cells}

Due to the different culture conditions with respect to our standard protocols in which amperometric signals are measured from cells adherent to a substrate layer (Carabelli et al., 2007; Marcantoni et al., 2009), we first tested the functionality of non-adherent (floating) mouse chromaffin cells by evaluating the size of voltage-gated $\mathrm{Ca}^{2+}$ channel currents and their coupling to the $\mathrm{Ca}^{2+}$-dependent secretion of catecholamines. Recordings were performed in the perforated-patch configuration to avoid the dialysis of the cell interior. By applying ramp commands from -60 to $+60 \mathrm{mV}$ (Fig. 3A), $\mathrm{Ca}^{2+}$ currents reached peak values between +10 and $+20 \mathrm{mV}$ (in $10 \mathrm{mM}$ external $\mathrm{Ca}^{2+}$ ) and had peak amplitudes comparable to those of mouse chromaffin cells cultured in standard conditions (mean current $256 \pm 47 \mathrm{pA} / \mathrm{pF}, n=5$ ). The shadowed area is arbitrarily drawn to highlight the $I / V$ relationship of voltage-gated $\mathrm{Ca}^{2+}$ channels that is typically recorded in the absence of functioning $\mathrm{Na}^{+}$ channels (Marcantoni et al., 2009). Depolarization-evoked secretion was measured by means of membrane capacitance increases (Fig. 3B). Test pulses of $100 \mathrm{~ms}$ duration at $+10 \mathrm{mV}$ caused a mean $3.5 \pm 0.4 \mathrm{pC} / \mathrm{pF} \mathrm{Ca}^{2+}$ influx and a corresponding $45.5 \pm 3.8 \mathrm{fF}$ capacitance increase $(n=5)$, in full agreement with measurements performed in $5 \mathrm{mM}$ external $\mathrm{CaCl}_{2}$ on cells adherent to a bottom plate (Marcantoni et al., 2009).

Also the entity of the readily releasable pool (RRP) was not significantly affected by these different culture conditions, suggesting that voltage-gated $\mathrm{Ca}^{2+}$ channels are functionally expressed and effectively coupled to the secretory machinery. The mean RRP was estimated by means of the double-pulse protocol shown in Fig. 3C $(85 \pm 6 \mathrm{fF} ; n=5)$, in agreement with values reported by Marcantoni et al. (2009). The two pulses (1 and 2) were delivered to produce the same quantity of $\mathrm{Ca}^{2+}$ charge. The RRP was determined by the equation $\left(\Delta C_{1}+\Delta C_{2}\right) /\left(1-\left(\Delta C_{2} / \Delta C_{1}\right)^{2}\right)$ (Gillis et al., 1996).

These preliminary experiments suggested that chromaffin cells, which were not stably attached to the adhesion layer but simply positioned over the NCD microelectrodes, possessed densities of voltage-gated $\mathrm{Ca}^{2+}$ channels and secretory activity comparable to the cells tightly adhering to the culture dish.

\subsection{Quantal event detection by planar NCD microelectrodes array}

In order to test whether the planar NCD device was able to detect quantal release from isolated chromaffin cells, catecholamine secretion was simultaneously assayed by NCD and CFEs (see Fig. 1B). Both NCD microelectrodes and CFE were polarized to $+650 \mathrm{mV}$ to detect maximal catecholamines oxidation currents. Even though synchronization of the acquired signals obtained by the two devices was not the purpose of this experiment, the simultaneous acquisition of secretion using different detectors was useful for testing the sensitivity of the NCD device (Fig. 4). Chromaffin cells were placed on the NCD microelectrode surface and bathed in a $\mathrm{KCl}$-free solution. If necessary, they were gently positioned on one of the four microelectrode wells by means of a glass patch-pipette controlled by a motor-driven micromanipulator (see Fig. 1B). The CFE was then positioned adjacent to the cell.

Sequences of amperometric spikes lasting 2-3 min started soon after cell stimulation with the $\mathrm{KCl}$ solution (arrows in Fig. 4) and were absent prior to the stimulation with $\mathrm{KCl}$ or when stimulating with $\mathrm{KCl}$-free solutions. Addition of the unspecific $\mathrm{Ca}^{2+}$ channels blocker $\mathrm{Cd}^{2+}(200 \mu \mathrm{M})$ completely prevented the secretory activity
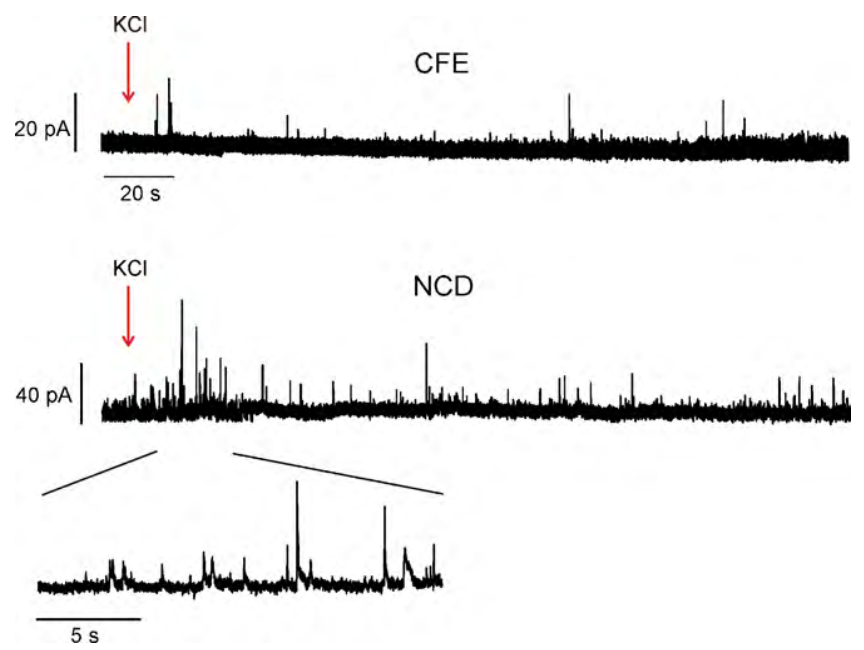

Fig. 4. Quantal secretory events from mouse chromaffin cells detected by the CFE and the planar boron-doped NCD device. Mouse chromaffin cells were stimulated by external $\mathrm{KCl}(30 \mathrm{mM})$ at the time indicated by the arrows. The inset shows representative amperometric bursts recorded by the NCD chip on a more expanded time scale. 

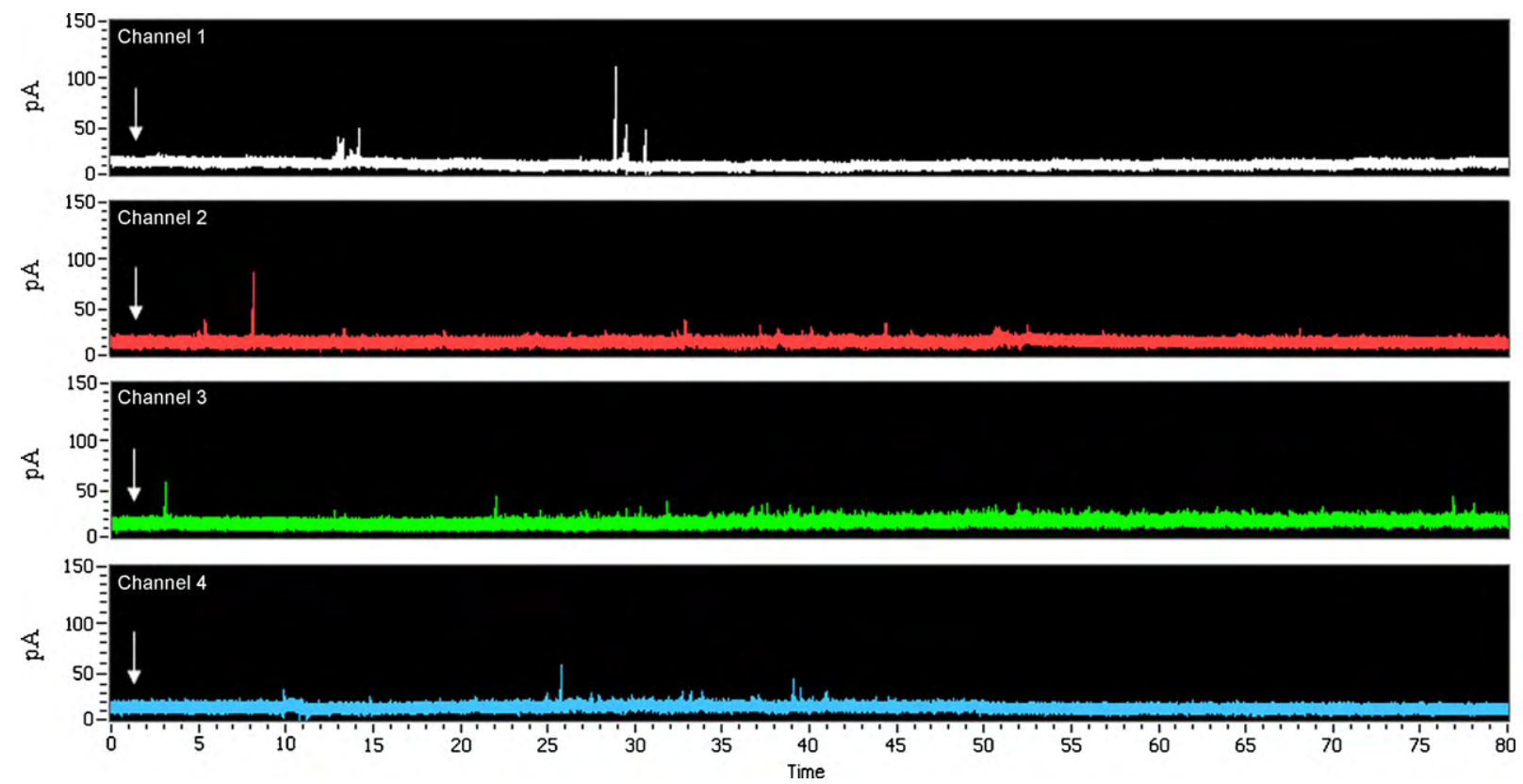

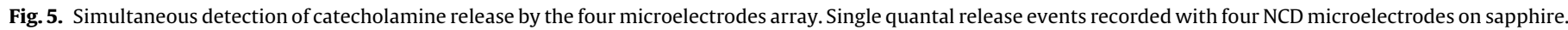

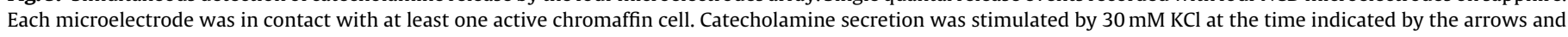
recorded for $80 \mathrm{~s}$.

(not shown). The amplitude of most visible amperometric spikes were well above the background noise (15 pA peak-to-peak) and varied from 10 to $120 \mathrm{pA}$. Spikes with amplitudes below $10 \mathrm{pA}$ were discarded from the analysis. Amperometric spikes recorded by the NCD microelectrode ( $n=127$ spikes) and the CFE ( $n=91$ spikes, $n=3$ cells) had comparable mean amplitude and quantity of charge: $24 \pm 5 \mathrm{pA}$ versus $21 \pm 3 \mathrm{pA}$, and $0.39 \pm 0.05 \mathrm{pC}$ versus $0.32 \pm 0.05 \mathrm{pC}$, respectively. The number of detected spikes by the NCD microelectrode was higher than the one with CFE, presumably because of the larger detection area of the NCD device with respect to the carbon fiber $\left(\sim 310 \mu \mathrm{m}^{2}\right.$ versus $\left.40 \mu \mathrm{m}^{2}\right)$, respectively. In conclusion, these experiments suggest that the NCD microelectrode array is able to detect amperometric signals with the same amplitude and signal-to-noise ratio as compared to the commonly used CFE.

\subsection{High-resolution of amperometric signals through the NCD multielectrode array}

The planar device has been designed with a simple four microelectrodes geometry array, in order to detect secretion by means of four independent channels. The responsiveness of the NCD by four different channels was achieved by positioning the chromaffin cells on the four electrode wells. Cells were stimulated by a $\mathrm{KCl}$ external solution, as indicated by the vertical arrows (Fig. 5), to depolarize the membrane and activate voltage-gated $\mathrm{Ca}^{2+}$-channels. Sequences of quantal events could be independently detected by the four output channels (ch1-ch4) with no visible correlations between the recorded signals. This excludes cross-talk between the four detecting NCD microelectrodes. In the case of Fig. 5, microelec-

(A)

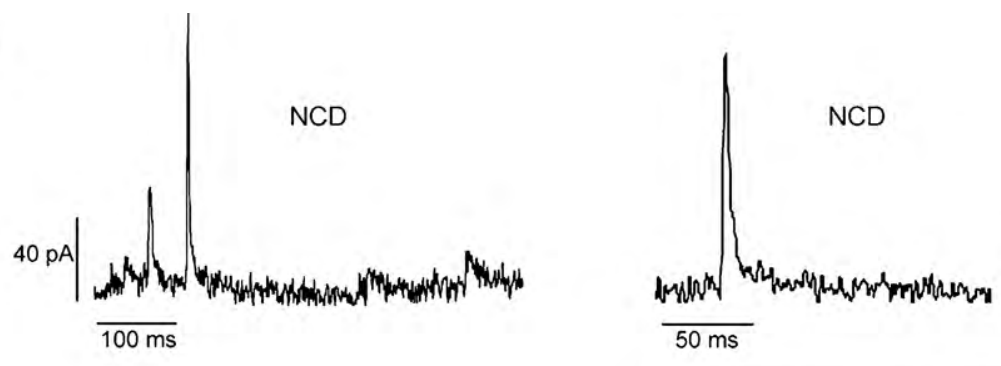

(B)

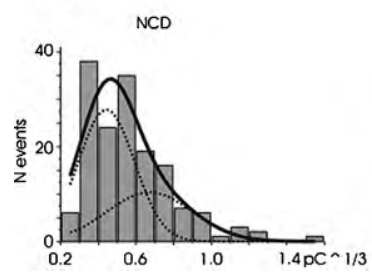

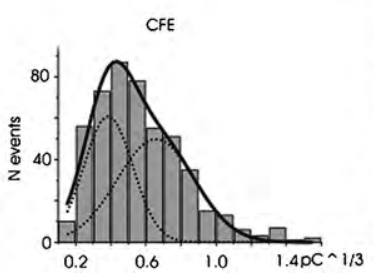

\begin{tabular}{|l|l|l|}
\hline & \multicolumn{1}{|c|}{ CFE } & \multicolumn{1}{c|}{ NCD } \\
\hline $\operatorname{Inax}(\mathrm{pA})$ & $33 \pm 6$ & $31 \pm 4$ \\
\hline $\mathrm{Q}(\mathrm{pC})$ & $0.35 \pm 0.07$ & $0.29 \pm 0.04$ \\
\hline $\mathrm{Q}^{1 / 3}\left(\mathrm{pC}^{13}\right)$ & $0.61 \pm 0.05$ & $0.57 \pm 0.02$ \\
\hline $\mathrm{m}(\mathrm{nA} / \mathrm{s})$ & $15 \pm 3$ & $15 \pm 3$ \\
\hline$t_{\mathrm{y} / 2}(\mathrm{~ms})$ & $7.1 \pm 0.8$ & $7.2 \pm 0.8$ \\
\hline $\mathrm{f}_{\mathrm{p}}(\mathrm{ms})$ & $9 \pm 3$ & $7.6 \pm 1.3$ \\
\hline
\end{tabular}

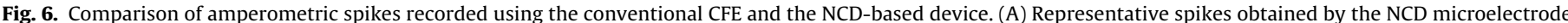

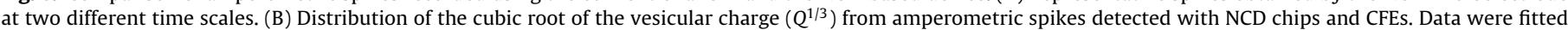

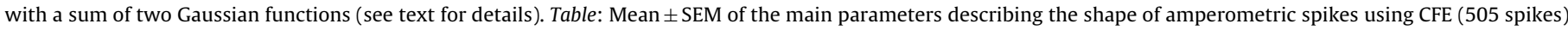
and NCD device (158 spikes). 
trode \#1 (white trace) showed two intense secretory activities in the form of bursts within $35 \mathrm{~s}$ and no activity afterward. Also electrode \#4 (blue trace) recorded large amperometric spikes up to $42 \mathrm{~s}$ and then remained silent, while electrodes \#3 (green trace) showed small amperometric signals up to the end of the recording interval (80 s), when all other channels were silent.

The four NCD microelectrode array allowed us to collect enough amperometric signals to undertake a detailed analysis of their time course and showed that the NCD array is equivalent to the CFE system. An enlarged view of some representative spikes recorded with the NCD array is given in Fig. 6. The analysis of the individual amperometric spikes was performed using Igor macros, as previously described (Marcantoni et al., 2009). Specifically, we measured five characteristic parameters of the spikes (Machado et al., 2001): the maximum oxidation current $\left(I_{\max }\right)$, the vesicular charge content $(Q)$, the rise-time of the spike $25-75 \%(m)$, the width at half-maximum $\left(t_{1 / 2}\right)$ and the time to the peak $\left(t_{\mathrm{p}}\right)$. Spike parameter values obtained by means of the NCD were then compared with those of classical CFEs. Notice that in the latter case chromaffin cells were plated in poly-ornithine treated plastic dishes (see Section 2). As indicated in the table of Fig. 6, we found no significant differences between the two recording conditions: the quantity of charge measured by CFEs and the planar device were not statistically different $(0.35 \pm 0.07 \mathrm{pC}$ versus $0.29 \pm 0.04 \mathrm{pC})$.

In agreement with previous data (Marcantoni et al., 2009), showing that mouse chromaffin cells possess a dual population of vesicles with different mean diameter $(d)$ (due to the spherical shape of chromaffin vesicle, $d$ is proportional to $Q^{1 / 3}$ ), the distribution of the cubic root of vesicle charge $\left(Q^{1 / 3}\right)$ was fitted with a double Gaussian function. By comparing data with NCD and CFE, we found that the two peaks were $0.44(55 \%) \mathrm{pC}^{1 / 3}$ and $0.67(45 \%) \mathrm{pC}^{1 / 3}$ with the NCD electrode, and $0.39(75 \%) \mathrm{pC}^{1 / 3}$ and $0.65(25 \%) \mathrm{pC}^{1 / 3}$ with the CFE. Correlation coefficient of the fit $\left(r^{2}\right)$ was good (0.98) for data with the CFE and somehow smaller for NCD data (0.85), due to lower number of statistical events. The form and amplitude of the amperometric spikes and the dual Gaussian distribution of vesicle diameter $\left(Q^{1 / 3}\right)$ were not significantly different when using CFEs and NCDs, suggesting that the two devices have the same sensitivity, low background noise and high-time resolution. The NCD biosensor has, however, the advantage that it can monitor secretory events simultaneously from multiple channels.

\section{Conclusions}

We fabricated a microelectrode array that takes advantage of nanocrystalline boron-doped diamond planar technology on sapphire to detect single $\mathrm{Ca}^{2+}$-dependent exocytotic events with high-time resolution, high degree of biocompatibility and reliability. The novel biosensor is chemically inert and has suitable optical transparency to allow cell manipulations under optical microscopes and the potential ability to be employed for fluorescence detection.

Given its wide hydrolysis potential window $(\sim 3 \mathrm{~V})$, the NCD multielectrode array can be also employed for monitoring electroactive species which are relevant for living cell functioning (e.g. $\mathrm{O}_{2}, \mathrm{CO}_{2}, \mathrm{H}^{+}$, NO) (Patel et al., 2008). Thanks to its chemical stability and wide hydrolysis potential window, the NCD microarray can be also employed for detection of other organic molecules besides catecholamines in both the cathodic and anodic regimes (Show et al., 2003). By differently polarizing the detecting electrodes, the chip has potential application for high-throughput screening of drugs and toxins acting on exocytosis and for measuring neurotransmitter release from in vitro neuronal networks or brain slices.

Finally, the combination of NCD diamond films with the sapphire substrate will allow the integration of the device into GaN electronics, widening the possibility of having with the same device amperometric and potentiometric measurements (Dipalo et al., 2009).

\section{Acknowledgments}

The work was supported by grants from the Ateneo ItaloTedesco and the Deutscher Akademischer Austausch Dienst (Vigoni program), the Regione Piemonte (grant no. D14-2005) and the Compagnia di SanPaolo (grant to the NIS and grant 2008.2191 to A.M).

\section{References}

Ariano, P., Lo Giudice, A., Marcantoni, A., Vittone, E., Carbone, E., Lovisolo, D., 2009 Biosens. Bioelectron. 24, 2046-2050.

Carabelli, V., Marcantoni, A., Comunanza, V., de Luca, A., Díaz, J., Borges, R., Carbone, E., 2007. J. Physiol. (London) 584, 149-165.

Chen, P., Xu, B., Tokranova, N.B., Feng, X., Castracane, J., Gillis, K.D., 2003. Anal. Chem. $75,518-524$

Chen, X., Gao, Y., Hossain, M., Gangopadhyay, S., Gillis, K.D., 2008. Lab Chip 8, 161-169.

Chow, R.H., von Rüden, L., Neher, E., 1992. Nature 356, 60-63.

Cui, H.-F., Ye, J.-S., Chen, Y., Chong, S.-C., Sheu, F.-S., 2006. Anal. Chem. 78, 6347-6355.

Dias, A.F., Dernick, G., Valero, V., Yong, M.G., James, C.D., Craighead, H.G., Lindau, M. 2002. Nanotechnology 13, 285-289.

Dipalo, M., Gao, Z., Scharpf, J., Pietzka, C., Alomari, M., Medjdoub, F., Carlin, J.-F., Grandjean, N., Delage, S., Kohn, E., 2009. Diamond Relat. Mater. 18, 884-889.

Gao, Y., Chen, X., Gupta, S., Gillis, K.D., Gangopdhyay, S., 2008. Biomed. Microdevices $10,623-629$

Gao, Z., Carabelli, V., Carbone, E., Colombo, E., Dipalo, M., Manfredotti, C., Pasquarelli, A., Feneberg, M., Thonke, K., Vittone, E., Kohn, E., 2009. IEEE Nanomed., Taiwan, 18-21 October.

Gao, Z., Carabelli, V., Carbone, E., Colombo, E., Demaria, F., Dipalo, M., Gosso, S., Manfredotti, Ch., Pasquarelli, A., Rossi, S., Xu, Y., Vittone, E., Kohn, E., 2010. Diamond Relat. Mater, doi:10.1016/j.diamond.2010.03.014, in press.

Gillis, K.D., Mossner, R., Neher, E., 1996. Neuron 16, 1209-1220.

Guillen, C., Herrero, J., 2005. Thin Solid Films 480-481, 129-132.

Hafez, I., Kisler, K., Berberian, K., Dernick, G., Valero, V., Yong, M.G., Craighead, H.G., Lindau, M., 2005. Proc. Natl. Acad. Sci. U.S.A. 102, 13879-13884.

Janischowsky, K., Ebert, W., Kohn, E., 2003. Diamond Relat. Mater. 12, 336-339.

Luong, J.H.T., Male, K.B., Glennon, J.D., 2009. Analyst 134, 1965-1979.

Machado, J.D., Morales, A., Gomez, J.F., Borges, R., 2001. Mol. Pharmacol. 60, 14-20.

Marcantoni, A., Carabelli, V., Vandael, D.H., Comunanza, V., Carbone, E., 2009. Pflugers Arch.: Eur. J. Physiol. 457, 1093-1110.

Minta, A., Kao, J.P., Tsien, R.Y., 1989. J. Biol. Chem. 264, 8171-8178.

Park, J., Galligan, J.J., Fink, G.D., Swain, G.M., 2006. Anal. Chem. 78, 6756-6764.

Parpura, V., 2005. Anal. Chem. 77, 681-686.

Patel, B.A., Galligan, J.J., Swain, G.M., Bian, X., 2008. Neurogastroenterol. Motil. 20 1243-1250.

Sen, A., Barizuddin, S., Hossain, M., Polo-Parada, L., Gillis, K.D., Gangopadhyay, S. 2009. Biomaterials 30, 1604-1612.

Show, Y., Witek, M.A., Sonthalia, P., Swain, G.M., 2003. Chem. Mater. 15, 879-888.

Sun, X., Gillis, K.D., 2006. Anal. Chem. 78, 2521-2525.

Titus, J.A., Haugland, R., Sharrow, S.O., Segal, D.M., 1982. Immunol. Methods 50 193-204.

Zhou, Y., Zhi, J., 2009. Talanta 79, 1189-1196.

Wightman, R.M., Jankowski, J.A., Kennedy, R.T., Kawagoe, K.T., Schroeder, T.J. Leszczyszyn, D.J., Near, J.A., Diliberto Jr., E.J., Viveros, O.H., 1991. Proc. Natl. Acad. Sci. U.S.A. 88, 10754-10758. 